\title{
Simulation of non-equilibrium electron transport in silicon quantum wires
}

\author{
Gerald Ossig $\cdot$ Ferdinand Schürrer
}

Published online: 16 May 2008

(C) Springer Science+Business Media LLC 2008

\section{Erratum to: J Comput Electron}

\subsection{7/s10825-008-0238-y}

We discovered a programming error in solving the Schrödinger-Poisson-Boltzmann system. Figures 2 and 4 are modified accordingly. From the results we can infer that the observed strong scattering effects are in good agreement with other approaches.

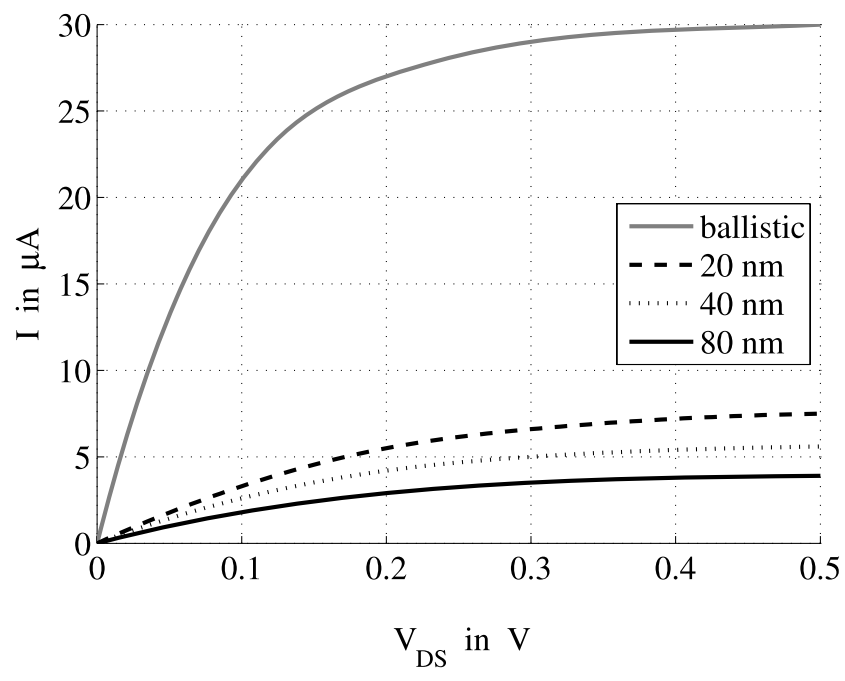

Fig. 2

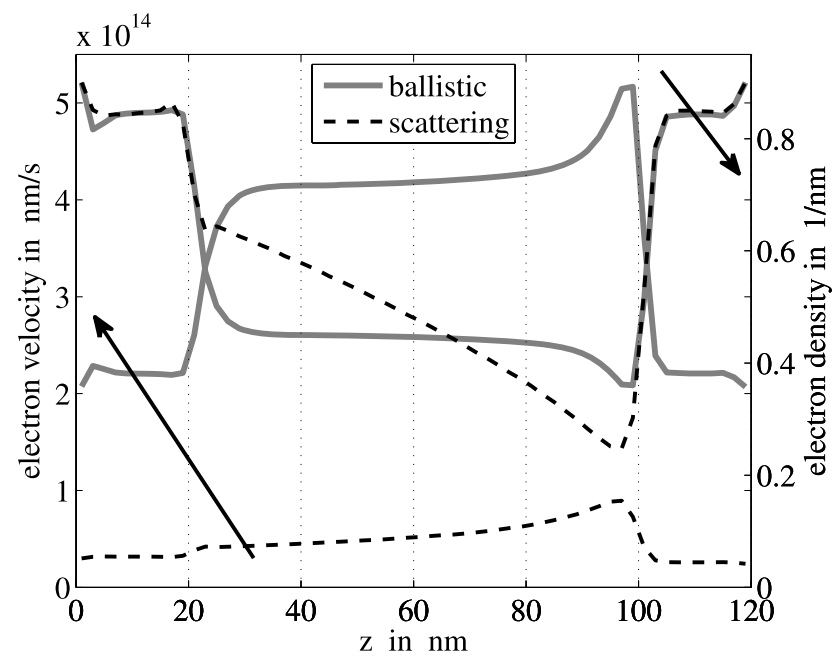

Fig. 4
The online version of the original article can be found under doi:10.1007/s10825-008-0238-y.

G. Ossig · F. Schürrer ( $₫)$

Institute of Theoretical and Computational Physics, TU Graz,

Petergasse 16, 8010 Graz, Austria

e-mail: schuerrer@itp.tugraz.at

G. Ossig

e-mail: ossig@itp.tugraz.at 